\title{
Making Decisions about Saving Energy in Compressed Air Systems using Ambient Intelligence and Artificial Intelligence
}

\author{
David Adrian Sanders \\ School of Engineering \\ University of Portsmouth \\ Portsmouth, PO1 3DJ, UK \\ david.sanders@port.ac.uk
}

\author{
David Charles Robinson \\ Cutting Tools Ltd \\ Castle Park Industrial Estate, \\ Oldham, OL1 3LN, UK \\ robinson@cut-tools.co.uk
}

\author{
Mohamed Hassan \\ School of Engineering \\ University of Portsmouth \\ Portsmouth, PO1 3DJ, UK \\ Mohamed.hassan@port.ac.uk

Malik Haddad
School of Engineering
University of Portsmouth
Portsmouth, PO1 3DJ, UK
malik.haddad@port.ac.uk

\author{
Alexander Gegov \\ School of Computing \\ University of Portsmouth \\ Portsmouth, PO1 3HE, UK \\ alexander.gegov@port.ac.uk \\ Nadia Ahmed \\ School of Engineering \\ University of Portsmouth \\ Portsmouth, PO1 3HE, UK \\ nadia.ahmed@port.ac.uk
}

\begin{abstract}
Compressed air systems are often the most expensive and inefficient industrial systems. For every 10 units of energy, less than 1 unit turns into useful compressed air. Air compressors tend to be kept fully on even if they are not (all) needed. The research proposed in this short paper will combine real time ambient sensing with Artificial Intelligence and Knowledge Management to automatically improve efficiency in energy intensive manufacturing. The research will minimise energy use for air compressors based on real-time manufacturing conditions (and anticipated future requirements). Ambient data will provide detailed information on performance. Artificial Intelligence will make sense of that data and automatically act. Knowledge Management will facilitate the processing of information to advise human operators on actions to reduce energy use and maintain productivity. The aim is to create new intelligent techniques to save energy in compressed air systems.
\end{abstract}

Keywords-Ambient; intelligence; energy; compressed air

\section{INTRODUCTION}

Concerns over climate change, coupled with volatility in oil markets have made energy efficiency a higher priority. The UK has a well-coordinated research community that is reducing energy in domestic and commercial buildings but the EPSRC [1], Energy Research Partnership [2] and Research Councils UK [3] have recognised that reducing energy use in industry is less well developed. UK Government has introduced stringent targets to achieve by 2020 , and more by 2050 . The UK manufacturing industry consumes $119,000 \mathrm{GWh}$ and produces the equivalent of 74 million tons of $\mathrm{CO} 2$. Equipments such as air compressors alone account for over $10 \%$ of UK industrial energy use (around 15,000 GWh) [4]. Industry needs to reduce both the financial and environmental costs of energy. The scientific community is well positioned to provide leadership in this area by introducing new technologies and paradigms to create a step improvement in manufacturing energy efficiency. Ambient information and knowledge gathered within a manufacturing environment are untapped resources for optimising energy use in real time and providing energy efficient manufacturing. The proposed approach described in this short paper is to investigate ambient sensing and artificial intelligence (AI) for manufacturing units [5] that interact with people and sensors to produce a detailed awareness of the process and environment, and to complement this with Knowledge Management (KM) systems to solve problems and provide suggestions; a holistic approach that takes advantage of all available relevant data / information [6].

Opportunities presented by intelligent sensor technologies will be exploited to create a step change in the approach to energy efficiency in manufacturing. This project will prove that the new concepts work for manufacturing units. The work focuses on air compressor-based manufacturing because that accounts for such a large proportion of UK industrial energy use, but the concepts proposed in this research will have the potential to be applied to other industries.

\section{BACKGROUND}

Ambient Intelligence uses seamless interaction between people and digital systems. It is reaching many sectors (consumer goods, healthcare and buildings) but industrial use is limited.

Ambient sensing information in combination with other classical sources can be used to optimise energy use. In the past such information has been difficult to obtain and that has led to cost intensive and incomplete monitoring and control. This research will create intelligent and interactive systems that work in real time and are more responsive to industrial needs and workers' actions and that become true enablers for optimising the energy efficiency of manufacturing systems. 
The latest manufacturing ideas are human-centred so that applying ambient sensing with $\mathrm{AI}$ and $\mathrm{KM}$ is promising. Issues need to be investigated and resolved to bring ambient sensing technology into industry, for example: trustworthy (wireless) sensing [7], understanding context [8], intelligent human computer interfaces [9] for the shop floor, security and safety [10], [11]. This research will contribute to this by creating new solutions using ambient sensing, AI [12] and KM [13].

Ambient sensing technology will involve using sensors in new ways to measure the performance of processes, such as the distribution of temperature across a machine using smart tags or real-time air flow in compressed air systems, which can provide data to compare with energy consumption data and be used to recognise problems. Ambient sensing technologies are still in an initial phase however, the application of ambient sensing in shop-floor manufacturing environments promises to bring advantages regarding: flexibility, reconfigurability and reliability. Ambient sensing technology is continually improving, reaching industrial standards, while, at the same time, prices of computing, sensors and tags are reducing, thereby making more extensive sensor systems affordable and practical. It is anticipated that industrial companies will introduce different ambient sensing technologies onto the shopfloor for configuration free manufacturing lines, etc. Manufacturing technology vendors will need to equip their machines, robots and tools with additional ambient sensing features and utilise the advantages within a shop-floor environment to provide new functions such as selfconfiguration and context-sensitivity to improve performance. This research will focus on the important potential of a combination of ambient sensing, AI and KM to optimise real time energy use in manufacturing.

The importance of KM has been recognized [14]-[16]. Knowledge needs to be applied efficiently [17]-[19] to enable energy to be utilised cost-effectively. To achieve that, several fundamental problems need to be addressed, including: human acceptance, how to correlate diverse kinds of knowledge and ways to use knowledge based on experience that might be ill structured and imperfect. Tacit knowledge and on-line capture and maintenance are not handled effectively in industry. e-KM tools have not satisfied industrial needs and so corporate knowledge continues to be managed heuristically and to rely on outdated methods. This project will address ways of automatically capturing knowledge by obtaining real time data from ambient sensors and changing that data into knowledge. This project offers a significant step forward in using KM to increase energy efficiency. Combining ambient sensing based knowledge from data capture with machine learning, deep reinforced learning and model-based reasoning for mapping higher-level knowledge will provide that step advance.

Energy efficiency actions in manufacturing are mostly carried out by consultants or specialist staff (both expensive) where data loggers and basic mathematical processing are normal and intelligence is provided by humans. Some intelligent energy efficiency systems can be found in largescale manufacturing but this project will provide automatic and intelligent systems that can be used more widely.
Information and help is available for companies to reduce energy consumption. For example, the European Commission EMAS Energy Efficiency Toolkit for SMEs [2] focuses on energy consumption and costs and the Carbon Trust (UK) provides an on-line checklist called the "Action Plan". However, the help tends to be general and limited.

Some systems are available but they are not real-time systems and they are aimed at power management and energy use in very large organisations. Some specific software tools are available such as MotorMaster+ for electrical motors, Pump System Assessment for pumps, and AirMaster+ for analysis of energy savings in air compressors, but they are not real-time systems and they are only used in a few large installations. There are several systems on the market but they are limited as none support real time energy optimisation.

Current practice in using analytical methods only covers basic statistics, bar charts, Pareto methods [3], [4] and energy flowcharts (Sankey diagrams), which can be useful in displaying the main areas of energy use. Power consumption curves can be useful in showing peaks in consumption, for example during machine start-ups. This can be reduced by using "soft-starts", where power is increased gradually at startup. Machinery can start and stop many times during a day and soft starting can result in large energy savings. In this proposed work, advanced methods including Experimental Design, Regression Analysis, Analysis of Variance and Surface Response techniques will be used to analyse energy consumption where combinations of different levels of different factors in the process are used and an optimum combination of variables can be estimated. These will be investigated and used to save energy in manufacturing processes.

Increasing complexity and demand for high productivity have led to growing applications of sensor networks to enable more reliable, timely, and comprehensive information from machines [4], [5]. Both classical measurement of energy consumption and modern sensory solutions with remote observation will be used. Benefits of adding a Bayesian network (or similar) to a sensor network will be investigated with a view to enabling the network to decide what constitutes an emergency or something to report to a monitoring supervisor [7]. Sensors might be able to provide more than just sensory information about energy usage. Based on information from several sensors, they may generate an aggregate which can suggest an appropriate course of action (turning a valve on or off, allowing a drive to come on at a particular time or delaying it for a while).

\section{NOVEL CONCEPT}

The concept is to combine ambient sensing, $\mathrm{AI}$ and $\mathrm{KM}$ to optimise energy efficiency. Recurrent neural networks and deep reinforced learning will be investigated with a view to using them with ambient data to provide detailed performance information for machine learning. KM will facilitate the processing of that information for a human computer interface. AI will ask questions and take automatic action. 


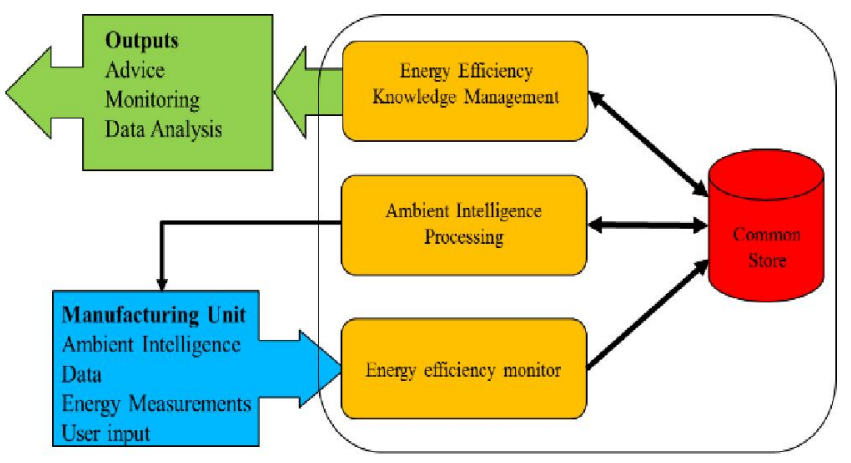

Fig. 1. Concept for combining ambient sensing, AI and KM.

Three input streams will come from the manufacturing unit (bottom left of Fig. 1): 1) Ambient intelligence data from sensors; 2) Energy measurements (measuring consumption); 3) Ambient sensing information from humans. Some intelligent processing of this input data will be carried out and the machine learning AI will act on it. Data will be stored within a recurrent neural network for the AI, alongside information and expert knowledge in a Common Store (red in Fig. 1). Energy efficiency KM will process the information to provide outputs (green / top left in Fig. 1).

Investigations into energy consumption will be combined with ambient sensing. For example, from interactions between operators and machines / processes, smart tags and measurements such as manufacturing line temperatures and production rates. The system will use deep reinforced learning to interactively provide advice, monitoring and data analysis. The aim is to create new intelligent techniques to save energy in compressed air systems. Objectives are to:

- Achieve a breakthrough in energy management.

- Use ambient sensing to monitor performance / environment.

- Create AI to evaluate performance and environment.

- Use knowledge management to reduce energy usage.

- Create a new interactive human computer interface.

\section{PROPOSED RESEARCH}

The authors are seeking funding for the proposed research.

\section{A. Data Streams}

Fig. 1 shows the three data streams from the manufacturing unit in blue (bottom left). Intelligent processing of this lower level data will be carried out using machine-learning techniques to make the data usable by the KM stage. The extent of such processing is to be investigated during the project.

Work will investigate hardware and circuit diagrams for existing systems to understand them and compare them with other options. Fuzzy Rule Based Systems will be created to interpret the input devices. Conditional statements, expressions and constructs will interpret smoothed data from input devices as those systems are suitable for specific knowledge.
A Fuzzy System will be created to identify ambient sensing information from interaction with humans (e.g. operator or supervisor) and interpret what they are doing. Fuzzy logic will deal with uncertainties generated by incomplete or partially corrupt data. Data and information will be deposited in the store. AI will take automatic action using a machine learning system (a recurrent neural network) and KM will process the information to give a range of energy efficiency outputs for the $\mathrm{HCI}$ and systems.

\section{B. Data Processing}

Measured data will be collected from energy consumption measuring and ambient sensing systems. This will be stored in a common store and re-used for analysis.

An important issue is to provide effective monitoring of processes to correlate data with energy consumption, as a basis for optimisation. Existing energy consumption sensors will be complemented with ambient sensors to measure interactions between operators and machines / processes and process measures such as line temperatures and production rates. The measurement systems will capture energy consumption and process / environmental data for intelligent event processing and computation. Sensor data will be converted into object models and used to determine any changes required. A Rule Based System (RBS) will consider information from sensors and select suitable fuzzy systems to provide input to a Decision-Making System (DMS). The DMS will compare outputs from the AI subsystems and suggest courses of action. The hybrid system combines desirable elements from different AI techniques. Their suggestions will be evaluated by the DMS. Ambient sensing data will typically come from: Manmachine interfaces; Smart tags and miniaturised information systems; Cameras and miniaturised optic; Micro-sensors to measure specific characteristics.

Data will be filtered and processed to provide a structured representation. Using a DMS to filter suggestions is like having a group of experts around a table. A Case Based Reasoning (CBR) system will provide confidence weightings for the different AI outputs so that the DMS can select an output. AI sub-systems will feed suggestions into the DMS. A set of problems will be created. The CBR system will then adapt solutions from previous problems to current problems. These solutions will be stored within a simple database representing experience. When a problem occurs that the system has not experienced, it will compare with previous cases and select one that is closest to the current problem. It will then act upon the solution given and update the database, depending upon the success or failure of the action. The CBR system will represent knowledge clearly to humans, but will also be able to learn from past examples. The DMS will arbitrate between the different AI systems when they fail to agree and different algorithms will be tested for providing balanced arbitration, including: Highest Confidence, Static and Dynamic Weighted, Summation of Confidences, Bonus Loading and Best / Worst. Using confidence values from the AI with dynamic weightings from the DMS, will improve decisions. A common store will be created to store the data and make it available. This will be the central source of information / knowledge. The research will consider the following modelling methodologies: ARIS, CIMOSA, GRAI/GIM, IEM, PERA and EN ISO 19440 to 
achieve an adequate solution to combine AI, KM \& ambient sensing, which will be flexible but also easy to understand. The store is in red on the right in Fig. 1.

\section{Ambient Intelligence Information}

Mapping will require the application and correlation of existing knowledge and new knowledge from other sources. The methodology will optimise energy use but it will provide an innovative approach to combining ambient sensing, AI and KM technology. It will address both technological and organisational aspects related to the research concepts and will serve as a set of guidelines for other researchers to further develop the approaches. The research will consider how to use information from ambient sensing and AI to optimise efficiency and how to organise collaboration between equipment and users for optimal energy use.

The information collected from ambient sensing and other sources will be turned into knowledge by adding context to the information. Information will be combined with existing knowledge and context models. The processing system will use information from ambient sensing and classical / legacy data sources (for example, Public Limited Companies). The AI will ask questions about the systems and take some automatic action. The Energy Efficiency Knowledge Management system will use the information to provide advice, monitoring and data analysis outputs. It will select and adapt parameter derivation models using the measured and processed data such as on-line diagnostics of energy related problems in the manufacturing unit and continuous improvement of energy consumption. The experimental system will interactively provide suggestions for energy efficiency improvements. The systems will use information middleware to provide measured data on-line. The middleware will allow the sharing of information / knowledge stored in the store and support the identification of relevant Energy efficiency knowledge in the store.

\section{Outputs}

Data analysis output will provide automatic analysis of historic data. Ambient sensing based upgrades to existing systems will be created, specific for each application. General solutions will be composed for several commonly used equipments such as motors, pumps and valves. Metric functions will be derived from measured data to identify the state of energy use and allow for effective management of energy use. Energy consumption and processes will be monitored and advice provided based on processed measurement data and knowledge gathered. Monitoring will include a prediction sub-module to identify energy related weak points (possibly before they occur).

\section{E. Experimentation}

A new experimental system will be created to test the methodology. This will test the soundness of the approaches through the research to ensure there is increased energy efficiency. These elements will include ambient sensing: sensors (temperature, line pressure, etc.), energy measurement, software, store and interfaces. Research will be tested in the University and at an industrial collaborator who will use the results to improve Energy efficiency of air compressor installations. They will provide a test bed and valuable data.
Investigations into the role of ambient sensing in monitoring manufacturing units and the format of the information space generated by the ambient sensing will be conducted. A new experimental system will be created to test the methodology. This will test the soundness of the approaches through the research to ensure there is increased energy efficiency. These elements will include ambient sensing: sensors (temperature, line pressure, etc.), energy measurement, software, store and interfaces.

\section{REFERENCES}

[1] EPSRC Research Area, Energy efficiency https://www.epsrc.ac.uk/research/ourportfolio/researchareas

[2] Research Councils UK International Review of Energy (2010) http://www.rcuk.ac.uk/publications/reports/energy2010

[3] "Energy innovation milestones to 2050". (2016). Energy Research Partnership report.

[4] Thiede (2012) Energy Efficiency in Manufacturing Systems, Springer.

[5] DC Robinson, DA Sanders, and E Mazharsolook, (2015), Ambient intelligence for optimal manufacturing and energy efficiency, Assembly Automation Journal, vol 35, no. 3, pp. 234-248.

[6] DC Robinson, DA Sanders, and E Mazharsolook, (2014), Sensor-based ambient intelligence for optimal energy efficiency, Sensor Review, vol 34, no. 2, pp. 170 - 181.

[7] A Yachir et al. (2016). Event-Aware Framework for Dynamic Services Discovery and Selection in the Context of Ambient Intelligence and Internet of Things. IEEE Trans on automation science and engineering 13 (1), pp: 85-102.

[8] D Stokic, U Kirchhoff and H Sundmaeker (2006). Ambient Intelligence in Manufacturing Industry: Control System Point of View, The 8th IASTED Conference on Control and Applications, CA Montreal.

[9] Ambient Intelligence E4 SME project. Revolution in Industrial Environments - Euro' Commission IST Prog' (05-08). Finding new technological \& organisational approaches.

[10] IST Advisory Group: Experience and Application Research: Involving Users in the Development of Ambient Intelligence; EU- 0904.

[11] L Snidaro and G Foresti (2007). Knowledge representation for ambient security, Expert Systems 24, Pages: 321-333.

[12] D Sanders (2009) Recognizing shipbuilding parts using artificial neural networks and Fourier descriptors. Proc of IMechE Part B-Journal of Engineering Manufacture, Volume: 223 Issue: 3 Pages: 337-342.

[13] D Sanders, YC Tan, I Rogers, GE Tewkesbury, (2009) An expert system for automatic design - for - assembly, Assembly Automation, Vol. 29 Issue: 4, pp.378-388.

[14] A Gegov, D Sanders, and B Vatchova (2017), 'Aggregation of inconsistent rules for fuzzy rule base simplification' International Journal of Knowledge-Based and Intelligent Engineering Systems, vol 21, no. 3, pp. 135-145..

[15] D Sanders, A. Gegov and D. Ndzi (2018), Knowledge-based expert system using a set of rules to assist a tele-operated mobile robot. Studies in Computational Intelligence, vol. 751, Springer, pp. 371-392.

[16] D Sanders 2017, Using self-reliance factors to decide how to share control between human powered wheelchair drivers and ultrasonic sensors. IEEE Transactions on Neural Systems and Rehabilitation Engineering, vol 25, no. 8, pp. 1221-1229.

[17] D Sanders, D Ndzi, S Chester and M Malik, (2017), Adjustment of teleoperator learning when provided with different levels of sensor support while driving mobile robots. Lecture Notes in Networks and Systems, vol. 16, Springer, pp. 548-558.

[18] D Sanders (2018) Non-model-based control of a wheeled vehicle pulling two trailers to provide early powered mobility and driving experiences, IEEE Trans on Neural Systems \& Rehab Eng, 26 (1), pp. 96 - 104.

[19] D Sanders, H Sanders, A Gegov, and D Ndzi, 92018) Rule-based system to assist a tele-operator with driving a mobile robot. Lecture Notes in Networks and Systems, vol. 16, Springer, pp. 599-615. 